\title{
Best Proximity Point Results for Some Contractive Mappings in Uniform Spaces
}

\author{
Victoria Olisama, ${ }^{1}$ Johnson Olaleru, ${ }^{2}$ and Hudson Akewe ${ }^{2}$ \\ ${ }^{1}$ Department of Mathematics, Adeniran Ogunsanya College of Education, Otto/Ijanikin, Lagos, Nigeria \\ ${ }^{2}$ Department of Mathematics, University of Lagos, Lagos, Nigeria
}

Correspondence should be addressed to Victoria Olisama; vicolisama@yahoo.com

Received 20 June 2016; Revised 22 December 2016; Accepted 26 February 2017; Published 26 April 2017

Academic Editor: Sivaguru Sritharan

Copyright (C) 2017 Victoria Olisama et al. This is an open access article distributed under the Creative Commons Attribution License, which permits unrestricted use, distribution, and reproduction in any medium, provided the original work is properly cited.

\begin{abstract}
We introduce the concept of $J_{a v}$-distance (an analogue of $b$-metric), $\phi_{p}$-proximal contraction, and $\phi_{p}$-proximal cyclic contraction for non-self-mappings in Hausdorff uniform spaces. We investigate the existence and uniqueness of best proximity points for these modified contractive mappings. The results obtained extended and generalised some fixed and best proximity points results in literature. Examples are given to validate the main results.
\end{abstract}

\section{Introduction}

The importance of fixed point theory emerges from the fact that it furnishes a unified approach and constitutes an important tool in solving equations which are not necessarily linear. A large number of problems can be formulated as nonlinear equations of the form $T(x)=x$, where $T$ is a selfmapping in some framework; see [1-4] and other references therein. Nevertheless, an equation of the type $T(x)=x$ does not necessarily possess a solution if $T$ happens to be a nonself-mapping. In this case, one seeks an appropriate solution that is optimal in the sense that $d(x, T(x))$ is minimum. That is, we resolve a problem of finding an element $x$ such that $x$ is in best proximity to $T(x)$ in some sense.

Best proximity point theorem analyzes the condition under which the optimisation problem, namely, $\inf _{x \in A} d(x$, $T x)$, has a solution. The point $x$ is called the best proximity point of $T: A \rightarrow B$, if $d(x, T x)=d(A, B)$, where $d(A, B)=$ $\inf \{d(x, y): x \in A ; y \in B\}$. Note that the best proximity point reduces to a fixed point if $T$ is a self-mapping.

A best proximity point problem is a problem of achieving the minimum distance between two sets through a function defined on one of the sets to the other.

The very popular best approximation theorem is due to Fan [5]. If $A$ is a nonempty compact subset of a Hausdorff locally convex topological vector space $B$ and $T: A \rightarrow B$ is a continuous mapping, then there exists an element $x \in A$ such that $d(x, T x)=d(A, T x)$. Fan's results are not without shortcomings; the best approximation theorem only ensures the existence of approximate solutions, without necessarily yielding an optimal solution. But the best proximity point theorem provides sufficient conditions that ensure the existence of approximate solutions which are also optimal.

Afterwards many authors such as Eldred and Veeramani [6] have derived extensions of Fan's Theorem and the best approximation theorems in many directions. Significant best proximity point results are in [7-11] and other references therein.

In fixed point theory, other spaces of study other than metric spaces have been used by different authors. Pseudometric spaces interestingly generalise metric spaces. One of the spaces in literature that generalises the metric and pseudometric spaces is the uniform space.

Weil [12] was the first to characterise uniform spaces in terms of a family of pseudometrics and Bourbaki [13] provided the definition of a uniform structure in terms of entourages. Aamri and El Moutawakil [14] gave some results on common fixed point for some contractive and expansive maps in uniform spaces and provided the definition of $A$ distance and $E$-distance. 
Also, Olatinwo [15] established some common fixed point theorems for self-mappings in uniform spaces by using the $A$ - and $E$-distances. Dhagat et al. [16] proved some common fixed point theorems for pairs of weakly and semicompatible mappings using $E$-distances in uniform spaces. Hussain et al. [17] apply the concept of cyclic $(\psi)$ contractions to establish certain fixed and common point theorems on a Hausdorff uniform space.

In another development, Geraghty [18] introduced the generalised contraction self-map using comparison functions.

Another useful result is by Karapinar and Erhan [19] who gave the definition of a $k$-contractive map for non-selfmappings and Karapinar [20] who established some results on best proximity points of $\psi$-Geraghty contractive non-selfmappings.

Also, Basha [21] gave some necessary and sufficient conditions to claim the existence of unique best proximity points for proximal contractions in metric spaces. Mongkolkeha et al. [22] introduced proximal cyclic contractions in metric spaces which are more general than the class of proximal contractions given by Basha [21].

Motivated by the results above, we develop the concept of $\phi_{p}$-proximal contraction and $\phi_{p}$-proximal cyclic contractions in uniform spaces and obtain the existence and uniqueness of best proximity points of these non-self-contractive mappings using $J_{a v}$-distance function.

\section{Preliminaries}

The following definitions are fundamental to our work.

Definition 1 (see [13]). A uniform space $(X, \Gamma)$ is a nonempty set $X$ equipped with a uniform structure which is a family $\Gamma$ of subsets of Cartesian product $X \times X$ which satisfy the following conditions:

(i) If $U \in \Gamma$, then $U$ contains the diagonal $\Delta=\{(x, x)$ : $x \in X\}$.

(ii) If $U \in \Gamma$, then $U^{-1}=\{(y, x):(x, y) \in U\}$ is also in $\Gamma$.

(iii) If $U, V \in \Gamma$, then $U \cap V \in \Gamma$.

(iv) If $U \in \Gamma$, and $V \subseteq X \times X$ which contains $U$, then $V \in \Gamma$.

(v) If $U \in \Gamma$, then there exists $V \in \Gamma$ such that whenever $(x, y)$ and $(y, z)$ are in $V$, then $(x, z)$ is in $U$.

$\Gamma$ is called the uniform structure or uniformity of $X$ and its elements are called entourages, neighborhoods, surroundings, or vicinities.

Definition 2 (see $[14])$. Let $(X, \Gamma)$ be a uniform space. A function $p: X \times X \rightarrow R^{+}$is said to be an

(a) A-distance if, for any $V \in \Gamma$, there exists $\delta>0$ such that if $p(z, x) \leq \delta$ and $p(z, y) \leq \delta$ for some $z \in X$, then $(x, y) \in V$;

(b) $E$-distance if $p$ is an $A$-distance and $p(x, y) \leq$ $p(x, z)+p(z, y), \forall x, y, z \in X$.

Another extension of a metric space is the $b$-metric space.
Definition 3 (see [2]). Let $X$ be a nonempty set and $s \geq 1$ be a given real number. A map $d: X \times X \rightarrow \mathbf{R}$ is said to be a $b$-metric if and only if, for all $x, y, z \in X$, the following conditions are satisfied:

(i) $d(x, y)>0$ with $x \neq y$ and $d(x, y)=0$ if and only if $x=y$.

(ii) $d(x, y)=d(y, x)$.

(iii)

$$
d(x, y) \leq s[d(x, z)+d(z, y)]
$$

The pair $(X, d)$ is called a $b$-metric space. If $s=1$, it becomes a metric space.

Examples in literature to show that $b$-metric is a generalisation of a metric space are in $[16,21]$.

Now, we introduce the concept of $J_{a v}$-distance.

Definition 4. Let $(X, \Gamma)$ be a uniform space. A function $p$ : $X \times X \rightarrow R^{+}$is said to be a $J_{a v}$-distance if

(i) $p$ is an $A$-distance,

(ii) $p(x, y) \leq s[p(x, z)+p(z, y)], \forall x, y, z \in X, s \geq 1$.

Note that the function $p$ reduces to an $E$-distance if the constant $s$ is taken as 1 .

Example 5. Let $(X, \Gamma)$ be a uniform space and let $d$ be a $b$ metric on $X$. It is clear that $\left(X, \Gamma_{d}\right)$ is a uniform space where $\Gamma_{d}$ is the set of all subsets of $X \times X$ satisfying $B_{\epsilon}=\{(x, y) \epsilon$ $\left.X^{2}: d(x, y)<\epsilon\right\}$ for some $\epsilon>0$. Moreover, if $\Gamma \subseteq \Gamma_{d}$, then $d$ is an $J_{a v}$-distance on $(X, \Gamma)$.

Also, the following definition is required.

Definition 6 (see $[13])$. Let $(X, \Gamma)$ be a uniform space and $p$ an A-distance on $X$

(a) If $V \in \Gamma,(x, y) \in V$, and $(y, x) \in V, x$ and $y$ are said to be $V$-close. A sequence $\left(x_{n}\right)$ is a Cauchy sequence for $\Gamma$ if, for any $V \in \Gamma$, there exists $N \geq 1$ such that $x_{n}$ and $x_{m}$ are $V$-close for $n, m \geq N$. The sequence $\left(x_{n}\right) \in X$ is a $p$-Cauchy sequence if for every $\epsilon>0$ there exists $n_{0} \in N$ such that $p\left(x_{n}, x_{m}\right)<\epsilon$ for all $n, m \geq N$

(b) $X$ is $S$-complete if for any $p$-Cauchy sequence $\left\{x_{n}\right\}$, there exists $x \in X$ such that $\lim _{n \rightarrow \infty} p\left(x_{n}, x\right)=0$.

(c) $f: X \rightarrow X$ is $p$-continuous if $\lim _{n \rightarrow \infty} p\left(x_{n}, x\right)=0$ implies $\lim _{n \rightarrow \infty} p\left(f\left(x_{n}\right), f(x)\right)=0$.

(d) $X$ is said to be $p$-bounded if $\delta_{p}(X)=\sup \{p(x, y)$ : $x, y \in X\}<\infty$.

To guarantee the uniqueness of the limit of the Cauchy sequence for $\Gamma$, the uniform space $(X, \Gamma)$ needs to be Hausdorff.

Definition 7 (see [13]). A uniform space $(X, \Gamma)$ is said to be Hausdorff if and only if the intersection of all the $V \in \Gamma$ reduces to the diagonal $\Delta$ of $X, \Delta=\{(x, x), x \in X\}$. In other words, $(x, y) \in V$ for all $V \in \Gamma$ implies $x=y$. 
A uniform structure $\Gamma$ defines a unique topology $\tau(\Gamma)$ on $X$ for which the neighborhoods of $x \in X$ are the sets $V(x)=$ $\{y \in X:(x, y) \in V\}, V \in \Gamma$.

$f: X \rightarrow X$ is $\tau(\Gamma)$ continuous if $\lim _{n \rightarrow \infty} x_{n}=x$ with respect to $\tau(\Gamma)$ implies $\lim _{n \rightarrow \infty} f\left(x_{n}\right)=f(x)$ with respect to $\tau(\Gamma)$.

Observe that all the above maps are self-mappings.

A large number of articles investigate non-self-contractive mappings on metric spaces. Some of these are given below.

Definition 8 (see [19]). Let $(X, d)$ be a metric space and $A$ and $B$ be nonempty subsets of $X$. A mapping $T: A \rightarrow B$ is said to be a $k$-contraction if there exists $k \in[0,1)$ such that

$$
d(T x, T y) \leq k d(x, y), \quad \forall x, y \in A .
$$

Definition 9 (see [23]). Let $A$ and $B$ be nonempty subsets of a metric space $(X, d)$ and let $T: A \cup B \rightarrow A \cup B$ such that $T: A \cup B \rightarrow A \cup B$,

(i) $T$ is cyclic if $T(A) \subseteq B$ and $T(B) \subseteq A$.

(ii) $T$ is called a cyclic contraction if

$$
\begin{aligned}
& d(T x, T y) \leq k d(x, y)+(1-k) d(A, B) \\
& \\
& \forall x \in A, \quad y \in B,
\end{aligned}
$$

for some $k \in[0,1)$.

(iii) $T: A \cup B \rightarrow A \cup B$ is called a cyclic $\phi$-contraction if $\phi:[0, \infty) \rightarrow[0, \infty)$ is a strictly increasing map

$$
\begin{aligned}
d(T x, T y) \leq d(x, y)-\phi(d(x, y))+ & \phi(d(A, B)), \\
& \forall x \in A, y \in B .
\end{aligned}
$$

Note that (4) becomes (3) with $\phi(q)=(1-k) q$ for all $q \geq 0$. But the converse is not true in general (see [23]).

Among the generalisations of the Banach contraction is the proximal contraction given by Basha in [21] and the proximal cyclic contraction in [22].

Definition 10 (see [21]). Let $(A, B)$ be a nonempty subset of a complete metric space $(X, d)$. A mapping $T: A \rightarrow B$ is said to be a proximal contraction if there exists a nonnegative real number $\alpha<1$ such that

$$
\begin{gathered}
d(u, T(x))=d(A, B) \\
d(v, T(y))=d(A, B) \\
\Downarrow \\
d(u, v) \leq \alpha d(x, y),
\end{gathered}
$$

for all $u, x, v, y \in A$.

$$
\begin{aligned}
& A_{0}=\{x \in A: p(x, y)=p(A, B) \text { for some } y \in B\}, \\
& B_{0}=\{y \in B: p(x, y)=p(A, B) \text { for some } x \in A\} .
\end{aligned}
$$

Basha [21] proved the following theorem.
Theorem 11 (see [21]). Let $A, B$ be two nonempty subsets of a complete metric space $(X, d)$. Suppose that $T\left(A_{0}\right)$ is nonempty and closed. Let $T: A \rightarrow B$ satisfy the following conditions:

(a) $T$ is a proximal contraction,

(b) $T\left(A_{0}\right) \subseteq B_{0}$.

Then there exists a point $x \in A$ such that $d(x, T(x))=d(A, B)$. Moreover, if $T$ is injective on $A$, then the point $x$ such that $d(x, T(x))=d(A, B)$ is unique.

Definition 12 (see [22]). Let $S: A \rightarrow B$ and $T: B \rightarrow A$. The pair $(S, T)$ is called a proximal cyclic contraction pair if there exists $\alpha \in[0,1)$ such that

$$
\begin{gathered}
d(a, S(x))=d(A, B) \\
d(b, T(y))=d(A, B) \\
\Downarrow \\
d(a, b) \leq \alpha d(x, y)+(1-\alpha) d(A, B),
\end{gathered}
$$

for all $a, x \in A, b, y \in B$.

Given nonempty subsets $A$ and $B$ of a uniform space $(X, \Gamma)$, we adopt the following notations and definitions used for metric spaces to the context of uniform spaces.

Definition 13. Let $S: A \rightarrow B$ and $g: A \rightarrow A$ be an isometry. The mapping $S$ is said to preserve the isometric distance with respect to $g$ if

$$
\begin{aligned}
p(S(g(x)), S(g(y)))=p(g(x), g(y)), & \\
& \forall x, y \in A .
\end{aligned}
$$

Definition 14. An element $x^{*}$ is called a best proximity point of a mapping $T: A \rightarrow B$ if it satisfies the condition that $p\left(x^{*}, T\left(x^{*}\right)\right)=p(A, B)=\inf \{p(x, y): x \in A ; y \in B\}$.

Now, we give the definition of $\phi_{p}$-proximal contraction and $\phi_{p}$-proximal cyclic contraction for non-self-mapping in uniform spaces.

Definition 15. Let $(A, B)$ be a pair of nonempty subsets of an $S$-complete Hausdorff uniform space $(X, \Gamma)$ such that $p$ is an $J_{a v}$-distance on $X$. A mapping $T: A \rightarrow B$ is said to be a $\phi_{p}$-proximal contraction if there exists a nondecreasing continuous weak comparison function $\phi: R^{+} \rightarrow R^{+}$ satisfying the following.

$\left(\mu_{1}\right)$ For each $t \in(0, \infty), 0<\phi(t)$ and $\phi(0)=0$,

$\left(\mu_{2}\right) \lim _{n \rightarrow \infty} \phi^{n}(t)=0, \forall t \in(0, \infty)$,

$\left(\mu_{3}\right) \phi(t)<t \forall t \in(0, \infty)$,

$\left(\mu_{4}\right) \sum_{n=0}^{\infty} \phi^{n}(t)$ converges for any $t$, such that $\forall j, l, k, m \in$ $A$, such that

$$
\begin{gathered}
p(j, T(k))=p(A, B) \\
p(l, T(m))=p(A, B) \\
\Downarrow \\
p(j, l) \leq \phi(p(k, m)) .
\end{gathered}
$$


Definition 16. Let $(A, B)$ be a pair of nonempty subsets of $S$ complete Hausdorff uniform space $(X, \Gamma)$ such that $p$ is an $J_{a v}$-distance on $X$. Suppose $S: A \rightarrow B$ and $T: B \rightarrow A$ are mappings. The pair $(S, T)$ is said to be a $\phi_{p}$-proximal cyclic contraction if there exists a nondecreasing continuous weak comparison function $\phi: R^{+} \rightarrow R^{+}$satisfying $\mu_{1}-\mu_{4}$ above, such that

$$
\begin{gathered}
p(j, S(k))=p(A, B) \\
p(l, T(m))=p(A, B) \\
\Downarrow \\
p(j, l) \leq \phi(p(k, m))+p(A, B)-\phi(p(A, B)),
\end{gathered}
$$

for all $j, k \in A$ and $l, m \in B$.

It is easy to see that a self-mapping that is a $\phi_{p}$-proximal contraction is a contraction. But a non-self $\phi_{p}$-proximal contraction is not necessarily a contraction map. If $\phi(w)=$ $\alpha w$ and $J_{a v}$-distance $p$ is replaced with a metric $d,(9)$ reduces to (6). Similarly, (8) reduces to (5). Also, (9) and (8) reduce to (2) if $A=B, S=T, \phi(w)=k w$ and if the $J_{a v}$-distance $p$ is replaced with a metric $d$, in the sense that $\Gamma=\left\{(x, y) \in X^{2}\right.$ : $d(x, y)<s \epsilon\}, s \geq 0$.

The following example shows that $E$-distance function $p$ is different from the metric distance function $d$. In fact, the $E$ distance function $p$ reduces to the metric distance function $d$ when $X$ is a metric space.

Example 17. Let $A=(-\infty, 0]$ and $B=[2,+\infty)$ be nonempty closed subsets of $X=R$ with the usual metric. Let $H: A \rightarrow$ $B$ be a mapping given by $H(x)=-4 / x$ and $u=-1, v=$ $0, x=-4, y=-2$ and let $\phi(x)=x / 3$. It is easy to see that $d(-1, H(-4))=d(A, B)=d(0, H(-2))=2$.

Clearly, $H: A \rightarrow B$ is not a $\phi$-proximal contraction; that is, $d(-1,0)>\phi(d(-4,-2))$.

$H$ has no best proximity point since there is no $x \in A$ such that $d(x, H(x))=2$.

Now, taking $u=x-2, x=u+2, x<u$. And consider $p$ defined as $p(x, y)=|2 x|$.

Clearly, $p(u, v) \leq \phi(p(x, y))$ for all $u, v, x, y \in A$. $H$ is a $\phi_{p}$-proximal contraction and -1 is the unique best proximity point of $H$.

The following Lemma, which is true for self-mappings (see Lemma 2.4 [23]) can be proved for non-self-mappings.

Lemma 18 (see [14]). Let $(X, \Gamma)$ be a Hausdorff uniform space and $p$ be an A-distance on $X$. Let $\left\{x_{n}\right\}_{n=0}^{\infty},\left\{y_{n}\right\}_{n=0}^{\infty}$ be arbitrary sequences in $X$ and $\left\{\alpha_{n}\right\}_{n=0}^{\infty},\left\{\beta_{n}\right\}_{n=0}^{\infty}$ be sequences in $R^{+}$converging to 0 . Then, for $x, y, z \in X$, the following holds:

(a) If $p\left(x_{n}, y\right) \leq \alpha_{n}$ and $p\left(x_{n}, z\right) \leq \beta_{n} \forall n \in N$, then $y=$ $z$. In particular, $p(x, y)=0$ and $p(x, z)=0$, and then $y=z$.

(b) If $p\left(x_{n}, y_{n}\right)=p(A, B)$ and $p\left(x_{n}, z_{n}\right)=p(A, B)$, then $y_{n}=z_{n}$.

(c) If $p\left(x_{n}, y_{n}\right) \leq \alpha_{n}$ and $p\left(x_{n}, z\right) \leq \beta_{n} \forall n \in N$, then, $\left(y_{n}\right)_{n=0}^{\infty}$ converges to $z$. (d) If $p\left(x_{n}, x_{m}\right) \leq \alpha_{n} \forall m>n$, then $\left\{x_{n}\right\}_{n=0}^{\infty}$ is a $p$-Cauchy sequence in $(X, \Gamma)$.

The major aim of this paper is to prove results similar to Theorem 11 above in uniform spaces and give the modification of results on proximal contractions in [22-24] in uniform spaces.

\section{Main Results}

We give the first theorem.

Theorem 19. Let $(A, B)$ be a pair of nonempty subset $X$ of an $S$ complete Hausdorff uniform space $(X, \Gamma)$ such that $p$ is an $J_{a v^{-}}$ distance on $X$ and is $A_{0} \neq \emptyset$. Suppose a map $F: A \rightarrow B$ is such that $F\left(A_{0}\right) \subset B_{0}$ is a $\phi_{p}$-proximal contraction. Then there exists a unique point $x^{*} \in A_{0}$ such that $p\left(x^{*}, F\left(x^{*}\right)\right)=p(A, B)$.

Proof. Let $x_{0} \in A_{0}$, since $A_{0} \neq \emptyset$ and $F\left(A_{0}\right) \subset B_{0}$. There exists $x_{1} \in A$ such that $p\left(x_{1}, F\left(x_{0}\right)\right)=p(A, B)$. Also, since $F\left(x_{1}\right) \in B_{0}$, there exists $x_{2} \in A_{0}$ such that $p\left(x_{2}, F\left(x_{1}\right)\right)=$ $p(A, B)$. Furthermore, we obtain the sequences $\left\{x_{n}\right\}$ and $\left\{x_{n+1}\right\}$ subsets of $A_{0}$ such that

$$
\begin{aligned}
& p\left(x_{n}, F\left(x_{n-1}\right)\right)=p(A, B), \\
& p\left(x_{n+1}, F\left(x_{n}\right)\right)=p(A, B), \quad \forall n \in N .
\end{aligned}
$$

We show that $\left\{x_{n}\right\}$ is a complete $p$-Cauchy sequence whose limit is the unique best proximity point of $F$. Since $F$ is a $\phi_{p^{-}}$ proximal contraction, from (10) and (11) we have

$$
p\left(x_{n}, x_{n+1}\right) \leq \phi\left(p\left(x_{n-1}, x_{n}\right)\right) \text {. }
$$

Thus by induction,

$$
p\left(x_{n}, x_{n+1}\right) \leq \phi^{n}\left(p\left(x_{0}, x_{1}\right)\right)
$$

for any $n=1,2, \ldots$.

Since $p$ is an $J_{a v}$-distance, we have $p\left(x_{n}, x_{m}\right) \leq$ $s\left[p\left(x_{n}, x_{n+1}\right)+\cdots+p\left(x_{m-1}, x_{m}\right)\right], s \geq 1$. Now for $r \geq 1$,

$$
\begin{aligned}
& p\left(x_{n}, x_{n+r}\right) \\
& \quad \leq s\left[\phi^{n}\left(p\left(x_{0}, x_{1}\right)\right)+\cdots+\phi^{n+r-1}\left(p\left(x_{0}, x_{1}\right)\right)\right] .
\end{aligned}
$$

Let $J_{n}=s \sum_{t=0}^{n} \phi^{t}\left(p\left(x_{0}, x_{1}\right)\right), n \geq 0$. Then

$$
p\left(x_{n}, x_{n+r}\right) \leq J_{n+r-1}-J_{n-1} .
$$

Suppose $p\left(x_{0}, x_{1}\right)>0$, and since $\phi$ is a weak comparison function, by Definition $15\left(\mu_{4}\right)$, it follows that

$$
\sum_{t=0}^{\infty} \phi^{t}\left(p\left(x_{0}, x_{1}\right)\right)<\infty .
$$

So there exists a $J \in[0, \infty)$ such that $\lim _{n \rightarrow \infty} J_{n}=J$. Then by (15),

$$
\lim _{n \rightarrow \infty} p\left(x_{n}, x_{n+r}\right)=0 .
$$


Repeating the same argument, we obtain $\lim _{n \rightarrow \infty} p\left(x_{n+r}\right.$, $\left.x_{n}\right)=0$.

Therefore, the sequence $\left\{x_{n}\right\}$ is a $p$-Cauchy in the $S$ complete space $(X, \Gamma)$. Hence there exists $x^{*} \in A_{0}$ such that

$$
\lim _{n \rightarrow \infty} p\left(x_{n}, x^{*}\right)=0
$$

since $A_{0}$ is closed. We prove that $x^{*}$ is the best proximity point of $F$; that is, $p\left(x^{*}, F\left(x^{*}\right)\right)=p(A, B)$.

Also, since $F\left(x_{0}\right) \subseteq B_{0}$ and $F\left(x^{*}\right) \subset B_{0}$, there exists an element $q \in A_{0}$ such that

$$
p\left(q, F\left(x^{*}\right)\right)=p(A, B)
$$

Using (19) and (11) and since $F$ is a $\phi_{p}$-proximal contraction,

$$
p\left(q, x_{n+1}\right) \leq \phi\left(p\left(x^{*}, x_{n}\right)\right)
$$

As $n \rightarrow \infty, p\left(q, x_{n+1}\right) \rightarrow 0$ since $p\left(x^{*}, x_{n}\right) \rightarrow 0$. Therefore, $x_{n} \rightarrow q$ and thus $q=x^{*}$. So from (19),

$$
p\left(x^{*}, F\left(x^{*}\right)\right)=p(A, B) .
$$

To guarantee the uniqueness of $x^{*}$, we show that $(X, \Gamma)$ is Hausdorff. Suppose there exists $y^{*}$ such that

$$
p\left(y^{*}, F\left(y^{*}\right)\right)=p(A, B) .
$$

By the $\phi_{p}$-proximal contraction $F$,

$$
p\left(x^{*}, y^{*}\right) \leq \phi\left(p\left(x^{*}, y^{*}\right)\right)<p\left(x^{*}, y^{*}\right),
$$

which implies $p\left(x^{*}, y^{*}\right)=0$. Similarly, $p\left(y^{*}, x^{*}\right)=0$. But by the second property of $J_{a v}$-distance,

$$
p\left(x^{*}, x^{*}\right) \leq s\left[p\left(x^{*}, y^{*}\right)+p\left(y^{*}, x^{*}\right)\right] \text {. }
$$

Hence,

$$
p\left(x^{*}, x^{*}\right)=0 \text {. }
$$

We conclude that $x^{*}=y^{*}$.

Corollary 20. Let $(X, d)$ be a complete metric space. Suppose $f: A \rightarrow A$ satisfies $d(f(x), f(y)) \leq k d(x, y), k \in(0,1)$; then $f$ has a unique fixed point.

Proof. Set $\phi(t)=k t, A=B$, and $\Gamma=\left\{(x, y) \in X^{2}: d(x, y)<\right.$ $\epsilon\}$ in Theorem 19, to obtain the result.

Corollary 21 (see [19]). Let $A$ and $B$ be two nonempty subsets of a complete metric space $(X, d)$. Suppose $f: A \rightarrow B$ satisfies $d(f(x), f(y)) \leq k d(x, y), k \in(0,1)$. Then $f$ has a unique best proximity point.

Proof. Set $\phi(t)=k t, j=f(m), l=f(k)$ and $\Gamma=\{(x, y) \in$ $\left.X^{2}: d(x, y)<\epsilon\right\}$ in Theorem 19 , to obtain the corollary.
Corollary 22 (see [21]). Let $A$ and $B$ be two nonempty subsets of a complete metric space $(X, d)$. Suppose $A_{0}$ is nonempty and closed and $T: A \rightarrow B$ satisfies the following conditions:

(a) $T$ is a proximal contraction,

(b) $T\left(A_{0}\right) \subseteq B_{0}$.

Then there exists a unique point $x^{*} \in A$ such that $d\left(x^{*}, T\left(x^{*}\right)\right)=d(A, B)$. Moreover, $\forall x^{*} \in A$, and there exists a sequence $\left\{x_{n}\right\} \subseteq A$ such that $d\left(x_{n+1}, T\left(x_{n}\right)\right)=d(A, B)$ for every $n \in N \cup\{0\}$ and $x_{n} \rightarrow x^{*}$.

Proof. Set $\phi(t)=k t$ and $\Gamma=\left\{(x, y) \in X^{2}: d(x, y)<\epsilon\right\}$ in Theorem 19.

Now, we establish some results of best proximity point for $\phi_{p}$-proximal cyclic contractions in uniform spaces.

Theorem 23. Let $(A, B)$ be a pair of nonempty closed subset $X$ of a p-bounded and S-complete Hausdorff uniform space $(X, \Gamma)$ such that $A_{0}, B_{0} \neq \emptyset$ and $p$ is an $J_{a v}$-distance on $X$. Let $F: A \rightarrow$ $B, G: B \rightarrow A$, and $h: A \cup B \rightarrow A \cup B$ satisfy the following conditions:

(i) the pair $(F, G)$ is a $\phi_{p}$-proximal cyclic contraction,

(ii) $F\left(A_{0}\right) \subseteq B_{0}, G\left(B_{0}\right) \subseteq A_{0}$,

(iii) $A_{0} \subseteq h\left(A_{0}\right)$ and $B_{0} \subseteq h\left(B_{0}\right)$,

(iv) $h$ is isometry.

Then there exist unique points $x \in A$ and $y \in B$ such that

$$
\begin{aligned}
p(h(x), F(x)) & =p(h(y), G(y))=p(x, y) \\
& =p(A, B) .
\end{aligned}
$$

Further, if $x_{0}$ is any fixed element in $A_{0}$ and $y_{0}$ is any fixed element in $B_{0}$, the sequences $\left\{x_{n}\right\}$ and $\left\{y_{n}\right\}$, defined by

$$
\begin{aligned}
& p\left(h\left(x_{n+1}\right), F\left(x_{n}\right)\right)=p(A, B), \\
& p\left(h\left(y_{n+1}\right), F\left(y_{n}\right)\right)=p(A, B),
\end{aligned}
$$

converge to the best proximity points $x$ and $y$, respectively.

Proof. Let $x_{0}$ be fixed element in $A_{0}$. Since $F\left(A_{0}\right) \subseteq B_{0}$ and $A_{0} \subseteq h\left(A_{0}\right)$, it follows that there exists an element $x_{1} \in A_{0}$ such that

$$
p\left(h\left(x_{1}\right), F\left(x_{0}\right)\right)=p(A, B) .
$$

Again, since $F\left(A_{0}\right) \subseteq B_{0}$ and $A_{0} \subseteq h\left(A_{0}\right)$, there exists an element $x_{2} \in A_{0}$ such that

$$
p\left(h\left(x_{2}\right), F\left(x_{1}\right)\right)=p(A, B) .
$$

Following the steps in the proof of Theorem 19, we can find $x_{n} \in A_{0}$ such that

$$
p\left(h\left(x_{n}\right), F\left(x_{n-1}\right)\right)=p(A, B) .
$$

By induction, one can determine an element $x_{n+1} \in A_{0}$ such that

$$
p\left(h\left(x_{n+1}\right), F\left(x_{n}\right)\right)=p(A, B) .
$$


Also, since $h$ is an isometry and by the $\phi_{p}$-proximity cyclic contraction using (30) and (31), it follows that, for each $n \geq 1$,

$$
\begin{gathered}
p\left(h\left(x_{n}\right), h\left(x_{n+1}\right)\right)=p\left(x_{n}, x_{n+1}\right) \leq \phi\left(p\left(x_{n-1}, x_{n}\right)\right)+p(A, B)-\phi(p(A, B)) \\
<\phi^{2}\left(p\left(x_{n-2}, x_{n-1}\right)\right) \\
\vdots \\
<\phi^{n}\left(p\left(x_{0}, x_{1}\right)\right) .
\end{gathered}
$$

Since $p$ is an $J_{a v}$-distance, we have $p\left(x_{n}, x_{m}\right) \leq s\left[p\left(x_{n}, x_{n+1}\right)+\right.$ $\left.\cdots+p\left(x_{m-1}, x_{m}\right)\right]$. Now for $q \geq 1$,

$$
\begin{aligned}
p\left(x_{n}, x_{n+q}\right) \leq & s \phi^{n}\left(p\left(x_{0}, x_{1}\right)\right)+\cdots \\
& +s \phi^{n+q-1}\left(p\left(x_{0}, x_{1}\right)\right) .
\end{aligned}
$$

Let $J_{n}=s \sum_{t=0}^{n} \phi^{t}\left(p\left(x_{0}, x_{1}\right)\right), n \geq 0$, and then

$$
p\left(x_{n}, x_{n+q}\right) \leq J_{n+q-1}-J_{n-1} .
$$

Next we show that $\left\{x_{n}\right\}$ is $p$-Cauchy in the $S$-complete space $X$; that is,

$$
\begin{aligned}
& \lim _{n \rightarrow \infty} p\left(x_{n}, x_{n+q}\right)=0, \\
& \lim _{n \rightarrow \infty} p\left(x_{n+q}, x_{n}\right)=0,
\end{aligned}
$$

for any $q \geq 1$.

Recall that

$$
p\left(x_{n+1}, F\left(x_{n}\right)\right)=p(A, B),
$$

if there exists $n_{0} \in N$ such that $x_{n_{0}+1}=x_{n_{0}}$, we are done, and $x_{n_{0}}$ is the required best proximity point of $F$. Thus we assume that $x_{n+1} \neq x_{n}$. have

Suppose $p\left(x_{0}, x_{1}\right)>0$. Now using Definition $15\left(\mu_{4}\right)$, we

$$
\sum_{t=0}^{\infty} \phi^{t}\left(p\left(x_{0}, x_{1}\right)\right)<\infty
$$

so there exists a $J \in[0, \infty)$ such that $\lim _{n \rightarrow \infty} J_{n}=J$.

Then by (35),

$$
\lim _{n \rightarrow \infty} p\left(x_{n}, x_{n+q}\right)=0 \text {. }
$$

Repeating the same argument, we obtain

$$
\lim _{n \rightarrow \infty} p\left(x_{n+q}, x_{n}\right)=0 .
$$

So the sequence $\left\{x_{n}\right\}$ is $p$-Cauchy in the $S$-complete space $(X, \Gamma)$.

Hence, $\left\{x_{n}\right\}$ converges to some element $x \in A$. Similarly, since $F\left(B_{0}\right) \subseteq A_{0}$ and $A_{0} \subseteq h\left(A_{0}\right)$, there exists a sequence $\left\{y_{n}\right\}$ such that it converges to some element $y \in B$ and from (31),

$$
p\left(h\left(y_{n+1}\right), G\left(y_{n}\right)\right)=p(A, B) .
$$

Since the pair $(F, G)$ is a $p$-proximal cyclic contraction and $h$ is isometry, using (31) and (41), we have

$$
\begin{aligned}
& p\left(h\left(x_{n+1}\right), h\left(y_{n+1}\right)\right)=p\left(x_{n+1}, y_{n+1}\right) \\
& \quad \leq \phi\left(p\left(x_{n}, y_{n}\right)\right)+p(A, B)-\phi(p(A, B)) .
\end{aligned}
$$

By (33), on taking limit as $n \rightarrow \infty$, we have

$$
p(x, y) \leq \phi(p(x, y))+p(A, B)-\phi(p(A, B)) .
$$

We show that $p(x, y)=p(A, B)$. Assume $p(x, y) \neq p(A, B)$, from (43), $p(x, y)<p(x, y)$, a contradiction. Hence,

$$
p(x, y)=p(A, B) .
$$

Thus, $x \in A_{0}$ and $y \in B_{0}$. Since $F\left(A_{0}\right) \subseteq B_{0}$ and $G\left(B_{0}\right) \subseteq A_{0}$, there exist $\tau \in A$ and $\eta \in B$ such that

$$
\begin{aligned}
& p(\tau, F(x))=p(A, B), \\
& p(\eta, G(y))=p(A, B) .
\end{aligned}
$$

Now, we show that $\tau=h(x)$ and $\eta=h(y)$.

Since $(F, G)$ is a $\phi_{p}$-proximal cyclic contraction, using (44) and (31) we have

$$
\begin{gathered}
p\left(\tau, h\left(x_{n+1}\right)\right) \leq \phi\left(p\left(x, x_{n}\right)\right)+p(A, B) \\
-\phi(p(A, B)) .
\end{gathered}
$$

Letting $n \rightarrow \infty$ in (46), $p(\tau, h(x))<p(x, x)$, and since $p$ is an $J_{a v}$-distance,

$$
p(\tau, h(x)) \leq s\left[p\left(x, x_{n}\right)+p\left(x_{n}, x\right)\right] .
$$

Again letting $n \rightarrow \infty$, we get $p(\tau, h(x)) \leq 0$ and so, $\tau=h(x)$. Therefore we have

$$
p(h(x), F(x))=p(A, B) .
$$

Similarly, we can obtain $\eta=h(y)$ and so,

$$
p(h(y), G(y))=p(A, B) .
$$


Thus, from (44), (48), and (49), we get

$$
\begin{aligned}
p(x, y) & =p(h(x), F(x))=p(h(y), G(y)) \\
& =p(A, B) .
\end{aligned}
$$

Next we prove the uniqueness of $x$ and $y$. Suppose that there exist $x_{a} \in A$ and $y_{a} \in B$ with $x \neq x_{a}$ and $y \neq y_{a}$ such that

$$
\begin{aligned}
& p\left(h\left(x_{a}\right), F\left(x_{a}\right)\right)=p(A, B), \\
& p\left(h\left(y_{a}\right), G\left(y_{a}\right)\right)=p(A, B) .
\end{aligned}
$$

Since $h$ is an isometry, and $F$ is a $p$-proximal cyclic contraction, using (48) and (51), we have

$$
\begin{aligned}
p\left(h(x), h\left(x_{a}\right)\right)= & p\left(x, x_{a}\right) \\
\leq & \phi\left(p\left(x, x_{a}\right)\right)+p(A, B) \\
& -\phi(p(A, B)) .
\end{aligned}
$$

$p\left(x, x_{a}\right)<p\left(x, x_{a}\right)$, a contradiction. Hence, $p\left(x, x_{a}\right)=$ 0 . Similarly, we show that $p\left(x_{a}, x\right)=0$. But since $p$ is a $J_{a v^{-}}$ distance, we have

$$
p\left(x_{a}, x_{a}\right) \leq s\left[p\left(x_{a}, x\right)+p\left(x, x_{a}\right)\right] .
$$

Therefore,

$$
p\left(x_{a}, x_{a}\right)=0 .
$$

Now we have $p\left(x_{a}, x_{a}\right)=0$ and $p\left(x, x_{a}\right)=0$. By Lemma 18(a), we conclude that $x_{a}=x$. Similarly, $y_{a}=y$.

Corollary 24 (see [17]). Let $(X, \Gamma)$ be a S-complete Hausdorff uniform space and $p$ an E-distance on $X$. Suppose $T: X \rightarrow X$ is a cyclic $\psi$-contraction such that $p(f(x), f(y)) \leq \psi(p(x, y))$, for all $x, y \in X$, where $\psi$ is a weak comparison function. Then $f$ has a unique fixed point.

Proof. The proof follows from Theorem 23 if $F=G, A=B$, and $J_{a v}$-distance is reduced to $E$-distance function.

Corollary 25 (see $[18])$. Let $(X, d)$ be a complete metric space and $T: X \rightarrow X$ be a Geraghty contraction satisfying $d(T x, T y) \leq \beta(d(x, y)) d(x, y)$ for each $x, y \in X$, where $\beta \in S$. Then $T$ has a unique fixed point.

Proof. The proof follows from Theorem 23 if $A=B, F=$ $G, \phi(t)=\beta(t)(t)$ and $p$ is a metric distance.

We give the following example to show that (9) generalises (6).

Example 26. Let $A, B \in X=R^{+}$such that $A=[1 / 4,1 / 2], B=$ $[3 / 4,1]$. Clearly, $d(A, B)=1 / 4$. Suppose $u=1 / 4, v=$ $3 / 4, x=1 / 2$, and $y=1$.
Let $S(x), T(x)$, and $p(x, y)$ be defined by

$$
\begin{aligned}
S(x) & =\frac{3 x}{2}, \\
T(x) & = \begin{cases}\frac{x}{4}, & x \in A ; \\
\frac{x}{2}, & \text { otherwise, }\end{cases} \\
p(x, y) & = \begin{cases}\frac{y}{3}, & x \in A, y \in B ; \\
\frac{2 y}{3}, & \text { otherwise. }\end{cases}
\end{aligned}
$$

Now, for $F\left(A_{0}\right) \subseteq B_{0}$ we obtain

$$
\begin{aligned}
& A_{0}=\{x \in A: p(x, y)=p(A, B)=1 / 4 \text { for some } y \in \\
& B\}=\{1 / 2\}, \\
& B_{0}=\{y \in B: p(x, y)=p(A, B)=1 / 4 \text { for some } x \in \\
& A\}=\{3 / 4\} .
\end{aligned}
$$

Now, (9) generalises (6) in the sense that

(1)

$$
\begin{aligned}
& d\left(\frac{1}{4}, S\left(\frac{1}{2}\right)\right)=d(A, B) \\
& d\left(\frac{3}{4}, T(1)\right)=d(A, B)
\end{aligned}
$$

$\Downarrow$

$$
d\left(\frac{1}{4}, \frac{3}{4}\right) \leq k d\left(\frac{1}{2}, 1\right)+(1-k) \frac{1}{4}
$$

for all $u, x \in A ; v, y \in B$.

$1 / 2 \leq k(1 / 2)+(1-k)(1 / 4)=(1 / 4)(k+1), k \in[0,1) \mathrm{a}$ contradiction.

Hence, (6) fails. $(S, T)$ is not a proximal cyclic contraction. We see that $(S, T)$ has no unique best proximity point since there is no $x \in A$ such that $d(u, S(u))=d(v, T(v))=1 / 4$.

But taking $u=x-1, x=u+1, x<u$,

(2)

$$
\begin{array}{r}
p(u, v) \leq \phi(p(x, y))-\phi(p(A, B))+p(A, B), \\
\forall u, x \in A, v, y \in B
\end{array}
$$

becomes $p(u, v)<p(x, y)$. $(S, T)$ is a $\phi_{p}$-proximal contraction. Clearly, $p(x, S(x))=p(y, T(y))=1 / 4$ and $1 / 2$ is the unique best proximity point of the pair $S$, while $3 / 4$ is the unique best proximity point of the pair $T$. Hence, (9) is different from (6).

We give the following examples to show that the $\phi_{p}$ proximal cyclic contraction is different from the Geraghty contraction.

Example 27. Consider the usual metric $(X, d), X=[0,1]$ and $d(x, y)=|x-y|$ and let $A=[0,1 / 10]$ and $B=[1 / 5,1]$. Obviously, $d(A, B)=1 / 10, A_{0}=1 / 10$ and $B_{0}=1 / 5, F\left(A_{0}\right) \subseteq$ $B_{0}$. Let $S: A \rightarrow B, T: B \rightarrow A$, be defined as $S(k)=5 k+1 / 5$, 
$T(m)=m / 10$ taking $j=1 / 50, k=1 / 25, i=2 / 5$ and $m=$ 1. Also, consider $\beta(x)=1-x$. And $p$ and $\psi$ are defined as follows:

$$
\begin{aligned}
& \psi(x)= \begin{cases}2 x, & x \in\left[0, \frac{1}{2}\right) ; \\
\frac{1}{5 x}, & x \in\left[\frac{1}{2}, 1\right] .\end{cases} \\
& p(x, y)= \begin{cases}\frac{y}{4}, & y \in\left[0, \frac{1}{2}\right], y>x ; \\
2 y, & y \in\left(\frac{1}{2}, 1\right] ; \\
1, & \text { otherwise. }\end{cases}
\end{aligned}
$$

We show that $S$ is not a Geraghty contraction $\psi(d(S(x)$, $S(y))) \leq \beta(\psi(d(j, k)))(\psi(d(j, k))) d(2 / 5,8 / 15)>(4 / 50) \times$ $(1 / 50)$, a contradiction. $S$ is not a Geraghty contraction.

We see that $S$ has no best proximity points since there is no $k \in A$ such that $d(k, S(k))=1 / 25$.

But $(S, T)$ is a $\phi_{p}$-proximal cyclic contraction. Clearly taking $m=2 l, p(j, S(k))=p(A, B)=p(l, T(m))$ implies $p(j, l) \leq \phi(p(k, m))+p(A, B)-\phi(p(A, B)), \forall j, k \in A$ and $l, m \in B$.

$(S, T)$ is a $\phi_{p}$-proximal cyclic contraction and $1 / 25$ is the unique best proximity point of $S$ while $2 / 5$ is the unique best proximity point of $T$.

\section{Conflicts of Interest}

The authors declare that they have no conflicts of interest.

\section{Authors' Contributions}

All authors contributed equally and significantly in writing this article. All authors read and approved the final manuscript.

\section{References}

[1] M. Abbas, A. R. Khan, and T. Nazir, "Coupled common fixed point results in two generalized metric spaces," Applied Mathematics and Computation, vol. 217, no. 13, pp. 6328-6336, 2011.

[2] I. A. Bakhtin, "The contraction mapping principle in almost metric spaces," Journal of Functional Analysis, vol. 30, pp. 2637, 1989.

[3] J. O. Olaleru, G. A. Okeke, and H. Akewe, "Coupled fixed point theorems for generalised $\phi$-mappings satisfying contractive condition of integral type on cone metric spaces," International Journal of Mathematical Modeling \& Computations, vol. 2, no. 2, pp. 87-98, 2012.

[4] J. O. Olaleru and H. O. Olaoluwa, "Common fixed points of four mappings satisfying weakly contractive-like condition in cone metric spaces," Applied Mathematical Sciences, vol. 7, no. 57-60, pp. 2897-2908, 2013.

[5] K. Fan, "Extensions of two fixed point theorems of F. E. Browder," Mathematische Zeitschrift, vol. 112, pp. 234-240, 1969.
[6] A. A. Eldred and P. Veeramani, "Existence and convergence of best proximity points," Journal of Mathematical Analysis and Applications, vol. 323, no. 2, pp. 1001-1006, 2006.

[7] A. Abkar and M. Gabeleh, "A note on some best proximity point theorems proved under P-property," Abstract and Applied Analysis, vol. 2013, Article ID 189567, 3 pages, 2013.

[8] A. P. Mihaela, "Best proximity point theorems for weak cyclic Kannan contractions," Filomat, vol. 25, no. 1, pp. 145-154, 2011.

[9] J. O. Olaleru and O. V. Olisama, "Coupled best proximity points of generalised Hardy-Rogers type cyclic $(\omega)$-contraction mappings," International Journal of Mathematical Analysis and Optimization, vol. 1, pp. 33-54, 2015.

[10] V. O. Olisama, J. O. Olaleru, and H. Olaoluwa, "Quadruple best proximity points of Q-cyclic contraction pair in abstract metric spaces," Asian Journal of Mathematics and Applications, vol. 2015, Article ID ama0206, p. 21, 2015.

[11] V.S. Raj, "A best proximity point theorem for weakly contractive non-self-mappings," Nonlinear Analysis: Theory, Methods \& Applications, vol. 74, no. 14, pp. 4804-4808, 2011.

[12] A. Weil, Sur les Espaces a Structure Uniforme et sur la Topologie Generale, vol. 551 of Actualités Scientifiques et Industrielles, Hermann, Paris, France, 1937.

[13] N. Bourbaki, Topologie Generale, Chapitre 1: Structures Topologiques, Chapitre 2: Structures Uniformes. Quatrieme Edition, Actualites Scientifiques et Industrielles, no. 1142, Hermann, Paris, France, 1965.

[14] M. Aamri and D. El Moutawakil, "Common fixed point theorems for E-contractive or E-expansive maps in uniform spaces," Acta Mathematica Academiae Paedagogicae Nyí Regyháziensis (New Series), vol. 20, no. 1, pp. 83-89, 2004.

[15] M. O. Olatinwo, "Some common fixed point theorems for self mappings in uniform space," Acta Mathematica Academiae Paedagogiace Nyíregyháziensis, vol. 23, no. 1, pp. 47-54, 2007.

[16] V. B. Dhagat, V. Singh, and S. Nath, "Fixed point theorems in uniform space," International Journal of Mathematical Analysis, vol. 3, no. 4, pp. 197-202, 2009.

[17] N. Hussain, E. Karapinar, S. Sedghi, N. Shobkolaei, and S. Firouzian, "Cyclic $\phi$-contractions in uniform spaces and related fixed point results," Abstract and Applied Analysis, vol. 2014, Article ID 976859, 7 pages, 2014.

[18] M. A. Geraghty, "On contractive mappings," Proceedings of the American Mathematical Society, vol. 40, pp. 604-608, 1973.

[19] E. Karapinar and M. Erhan, "Best proximity point on different type contractions," Applied Mathematics \& Information Sciences, vol. 5, no. 3, pp. 558-569, 2011.

[20] E. Karapinar, "On best proximity point of $\psi$-Geraghty contractions," Fixed Point Theory and Applications, vol. 2013, article 200, 2013.

[21] S. Sadiq Basha, "Best proximity points: optimal solutions," Journal of Optimization Theory and Applications, vol. 151, no. 1, pp. 210-216, 2011.

[22] C. Mongkolkeha, Y. J. Cho, and P. Kumam, "Best proximity points for Geraghty's proximal contraction mappings," Fixed Point Theory and Applications, vol. 2013, article 180, 2013.

[23] M. A. Al-Thagafi and N. Shahzad, "Convergence and existence results for best proximity points," Nonlinear Analysis: Theory, Methods \& Applications, vol. 70, no. 10, pp. 3665-3671, 2009.

[24] W. A. Kirk, S. Reich, and P. Veeramani, "Proximinal retracts and best proximity pair theorems," Numerical Functional Analysis and Optimization, vol. 24, no. 7-8, pp. 851-862, 2003. 


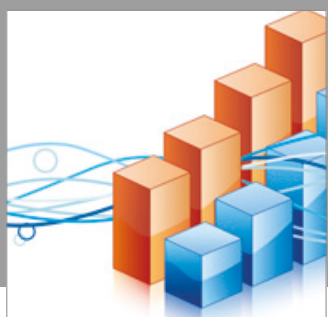

Advances in

Operations Research

vatersals

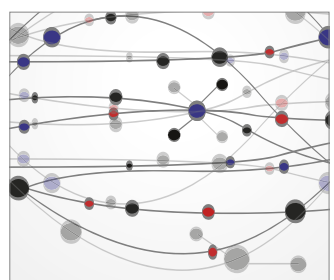

\section{The Scientific} World Journal
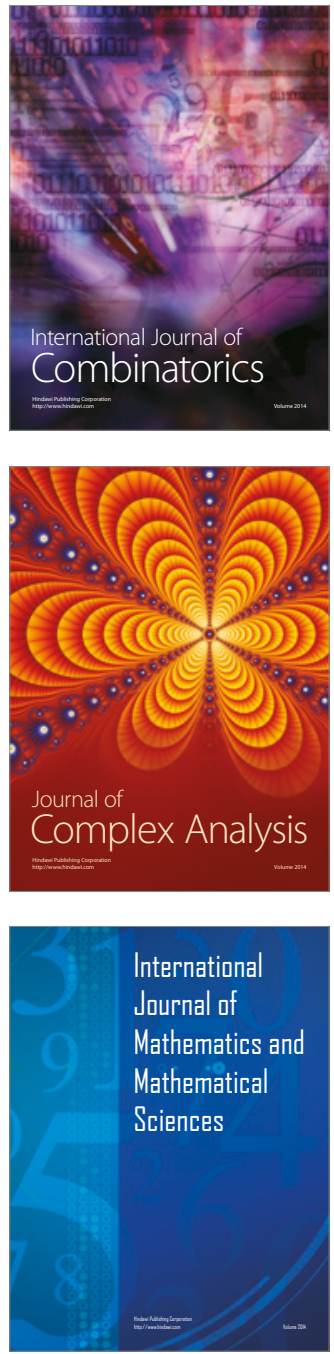
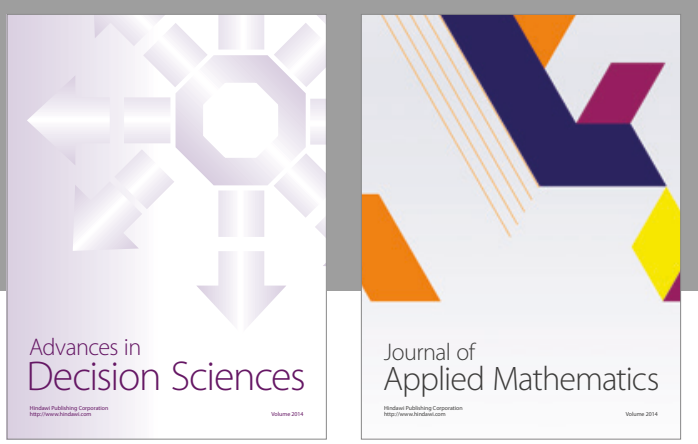

Algebra

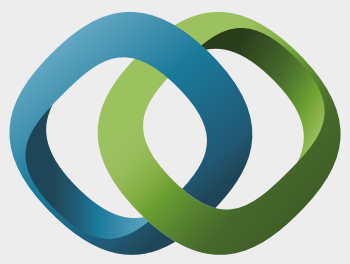

\section{Hindawi}

Submit your manuscripts at

https://www.hindawi.com
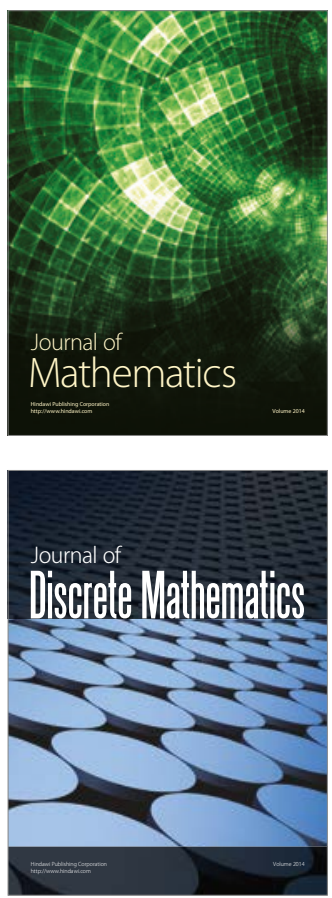

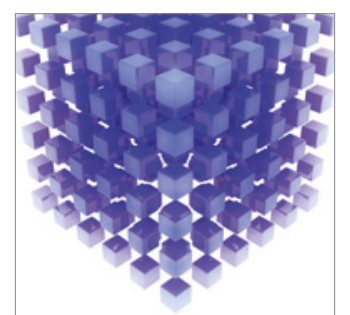

Mathematical Problems in Engineering
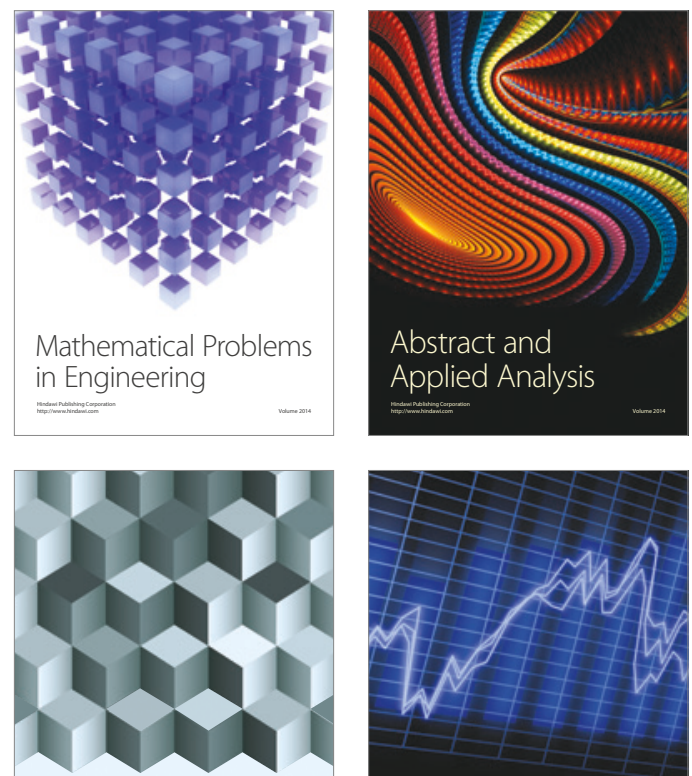

Journal of

Function Spaces

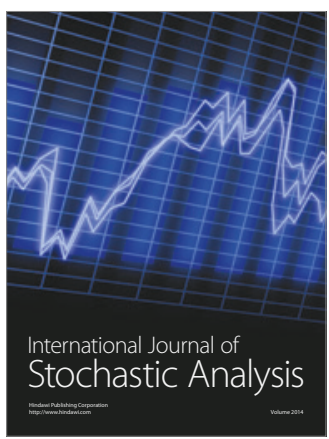

Probability and Statistics
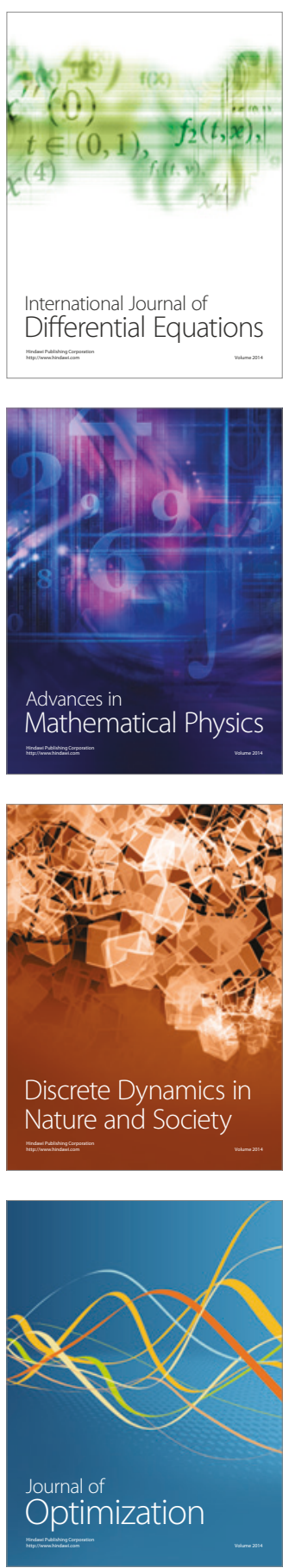